\title{
Effects of sample geometry on the dynamics and configurations of vortices in mesoscopic superconductors
}

\author{
Sangbum Kim, John Burkardt, Max Gunzburger, and Janet Peterson \\ School of Computational Science, Florida State University, Tallahassee, Florida 32306, USA \\ Chia-Ren $\mathrm{Hu}$ \\ Center for Theoretical Physics, Department of Physics, Texas A\&M University, College Station, Texas 77843, USA
}

(Received 20 March 2007; revised manuscript received 31 May 2007; published 16 July 2007)

\begin{abstract}
A computational study of mixed states in mesoscopic type-II superconducting cylinders is presented. The dependence of transient behaviors and steady-state configurations on the value of the applied magnetic field is examined as are the effects of sample size and cross-sectional shape on vortex nucleation and penetration. As is well known, more vortices enter the sample as the sample size grows. It is also found that if a small indentation is made on the sample boundary, vortices can be made to enter the system one by one from the tip of the indentation. An efficient scheme to determine, for any applied field, the equilibrium vortex configuration in mesoscopic samples in a way that is not constrained by sample symmetries, is devised and demonstrated.
\end{abstract}

DOI: 10.1103/PhysRevB.76.024509

\section{INTRODUCTION}

Recent advances in microfabrication and experimental techniques have drawn much attention to mesoscopic superconductors. Important fundamental and technological questions concerning magnetic properties and phase transitions in such small, bounded systems need to be considered. The dynamics of magnetic vortices plays a key role in understanding the magnetization process of type-II superconductors. As the size of the sample considered is reduced, the interaction between vortices and sample surfaces becomes increasingly important as does the sample shape.

Among the several phenomenological theories that have been developed for describing the magnetic behavior of superconductors, one popular choice is the Ginzburg-Landau theory. ${ }^{1,2}$ Its time-dependent extension is referred to as the time-dependent Ginzburg-Landau (TDGL) theory. ${ }^{3}$ Our computational study is based on finite-element discretizations of the TDGL equations. Such methods are well known to provide a powerful computational tool for the approximate solution of partial differential equations; they are particularly well suited for samples having complex shapes. See, e.g., Refs. 4 and 5 for an introduction to finite-element discretizations of the Ginzburg-Landau model. In this paper, we examine the effects of surface defects and sample geometry on the vortex dynamics using computational results obtained by using a finite-element method applied to the TDGL equations.

Several studies ${ }^{6-11}$ have been devoted to the magnetization of a mesoscopic superconductor without pinning centers. Calculations on the superconducting state in mesoscopic, type-I, superconducting thin films have, in most cases, found transitions between giant vortex states of different circulation quantum numbers $L$, with some multivortex states occasionally appearing as thermodynamically stable states, but mostly as metastable states. Another study ${ }^{12}$ of both type-I and type-II mesoscopic triangular cylinders has shown that a vortex-antivortex molecule appears only if the sample is type I. Only one field value for which $L=2$ is favored was considered; how changes in the value of the field affect the
PACS number(s): 74.78.Na, 74.25.Qt, 74.25.Op

configuration of vortices was not considered. Further studies $^{13}$ of the mixed state in a mesoscopic type-II superconducting cylinder revealed that the system passes through nearly metastable intermediate configurations as it seeks the one that minimizes the Gibbs free energy; the steady-state configuration was consistent with the square symmetry of the sample. In this study, vortices were "seeded" into the sample; i.e., at the beginning of the calculations, a given number of vortices were introduced into the sample at random positions and then were allowed to relax into more energetically favorable positions. The values of the Gibbs free energies of the final steady states that evolved from initial configurations having different numbers of vortices were used to determine the equilibrium vortex configuration in the mesoscopic sample. Note that in this study, vortices were "artificially" introduced into the sample, as opposed to allowing them to nucleate on their own.

For type-II superconductors, ${ }^{14}$ the magnetic field, in the form of singly quantized vortices, penetrates into a superconducting sample through its boundary. Circulating around each vortex is a supercurrent that confines a single quantum of magnetic flux within it. Thus, the penetration of the magnetic field into a superconductor in the form of quantized vortices is greatly affected by the interaction of the vortices with the sample surface and results in an energy barrier, which is manifest in the hysteretic behavior of the system even without pinning centers. Due to the surface barrier, the lowest critical field for which penetration occurs, denoted by $H_{p}$ (and referred to as the superheating field for vortex entry), is higher than the first critical field $H_{c 1}$ for samples having no boundaries. The effects of sample surfaces on the first vortex entry were considered in a number of studies ${ }^{15-17}$ for bulk superconductors. It was shown that vortices start penetrating into bulk type-II superconductors at a critical field $H_{p}$ that is close to the thermodynamic critical field $H_{c}$ but larger than $H_{c 1}$. In another study, ${ }^{18}$ a version of the TDGL equations that accounts for thermal fluctuations was used to computationally evaluate the effect of the surface barrier on magnetic flux penetration for a square, type-II superconducting sample of size $256 \xi \times 256 \xi$, where $\xi$ denotes 
the coherence length. It was found that the superheating field $H_{p}$ is a decreasing function of temperature and also of the degree of sample shape deformation. The surface barrier was also found to be suppressed by surface irregularities which were modeled by randomly distributed surface defects of the size of $\lambda$, the magnetic penetration depth. Yet another version of TDGL theory, consisting of the time-dependent GinzburgLandau equation for the order parameter and an equation for the scalar potential arising from the equation of continuity, was used to study square mesoscopic superconducting samples of sizes small in comparison with the magnetic penetration depth to obtain vortex states and magnetization curves. ${ }^{19}$ A small defect (normal metal) was introduced on the boundary of the samples to disturb the fourfold symmetry of their numerical scheme (explicit Euler), leaving the order parameter and current distribution almost undisturbed. This way they could obtain the branches on the magnetization curve with an odd total vorticity besides the even vorticity branches which they could already obtain without introducing any defects into the sample. Although one steady-state solution was found for each and every allowed vortex number, the equilibrium solution having minimum energy at any given magnetic field was not explicitly sought. Also, they used larger defects to examine the stability of a maximally symmetric vortex configuration with four vortices on the diagonals and one antivortex at the center of a square sample and showed that such a vortex state is very sensitive to any perturbation of the sample symmetry by defects or fluctuations. The antivortex was easily attracted off its original position to annihilate with a vortex by the introduction of a symmetry-breaking defect at the sample boundary. The TDGL equations (without thermal fluctuations) were also used $^{20}$ to study the surface barrier for both type-I and type-II mesoscopic superconducting samples. The dependence of the superheating field $H_{p}$ on the Ginzburg-Landau parameter $\kappa$ and on the boundary conditions was investigated. Multiple penetration fields $H_{p, i}=H_{p, 1}, H_{p, 2}, H_{p, 3}, \ldots$, were observed in the magnetization curves and were interpreted using an extended Bean-Livingston surface barrier model, assuming that $i-1$ vortices were already inside the sample. The effect of rectangular-shaped surface defects on the magnetic field for the first vortex entry using TDGL theory for isotropic, bulk type-II superconductors has also been studied. ${ }^{21}$ It was shown that the first-vortex-entry field decreases monotonically with growing lengths and decreasing widths of the defect. We note that the influence of small-size $(\ll \lambda)$ and largesize $(\gg \lambda)$ surface defects on the value of $H_{p}$ for bulk type-II superconductors has been studied ${ }^{22-25}$ using the London model.

This article is organized as follows. In the next section, the TDGL equations and the computational approach used are briefly discussed. In Sec. III, we study the magnetization process for type-II, cylindrical, mesoscopic, superconducting samples having different cross-sectional shapes and sizes. We find that whenever a sample has perfect symmetry, vortices nucleate at the boundary in multiples of the symmetry number and that more vortices enter the sample as its size grows. However, if a small indentation in the shape of a sliver is made at the sample boundary, vortices enter the system one by one from the tip of the indentation. In Sec. IV, a novel strategy for finding equilibrium states having an arbitrary number of vortices is presented. Steady-state vortex configurations are first created taking advantage of the oneby-one vortex nucleation feature of indented samples. These vortex states are then relaxed to new steady states after removal of the indentation. By comparing the Gibbs free energies of the steady states as a function of the number of vortices, the equilibrium state is determined. Finally, in Sec. V, a summary of the results of the article and concluding remarks are provided.

\section{TIME-DEPENDENT GINZBURG-LANDAU MODEL}

The TDGL equations are given by ${ }^{26}$

$$
\gamma\left(\hbar \frac{\partial \psi}{\partial t}+i e_{s} \Phi \psi\right)+\frac{1}{2 m_{s}}\left(i \hbar \boldsymbol{\nabla}+\frac{e_{s}}{c} \mathbf{A}\right)^{2} \psi+\alpha \psi+\beta|\psi|^{2} \psi=0
$$

and

$$
\begin{aligned}
\nu\left(\frac{1}{c} \frac{\partial \mathbf{A}}{\partial t}+\boldsymbol{\nabla} \Phi\right)+\frac{c}{4 \pi} \boldsymbol{\nabla} \times \boldsymbol{\nabla} \times \mathbf{A}+\frac{e_{s}^{2}}{m_{s} c}|\psi|^{2} \mathbf{A} \\
+i \frac{e_{s} \hbar}{2 m_{s}}\left(\psi^{*} \boldsymbol{\nabla} \psi-\psi \boldsymbol{\nabla} \psi^{*}\right)=0,
\end{aligned}
$$

where $(\cdot)^{*}$ denotes complex conjugation. The variables in the model are $\psi$, the complex-valued order parameter; $\Phi$, the scalar-valued electric potential; and $\mathbf{A}$, the vector-valued magnetic potential. The derived variables of physical interest include the induced magnetic field $\mathbf{h}=\boldsymbol{\nabla} \times \mathbf{A}$, the density of superconducting charge carriers $|\psi|^{2}$, and the supercurrent density $\mathbf{j}_{s}=-\frac{i e_{s} \hbar}{2 m_{s}}\left(\psi^{*} \nabla \psi-\psi \nabla \psi^{*}\right)-\frac{e_{s}^{2}}{m_{s} c}|\psi|^{2} \mathbf{A}$. In the above, $e_{s}$ denotes the "effective charge" of a Cooper pair which is twice the charge of an electron; $m_{s}$ its "effective mass," which, although it can be selected arbitrarily, is usually chosen to be twice the mass of an electron; $c$ is the speed of light; and $\hbar=h / 2 \pi$, where $h$ is Planck's constant.

For simplicity, we assume that the region exterior to the sample is a vacuum so that, along with the TDGL equations (1) and (2), we have that ${ }^{1,3}$

$$
\mathbf{n} \cdot\left(i \hbar \nabla+\frac{e_{s}}{c} \mathbf{A}\right) \psi=0
$$

at the boundary of the sample, where $\mathbf{n}$ denotes the unit normal pointing out of the sample. We also only consider cylindrical samples and constant applied magnetic fields $\mathbf{H}_{\text {ext }}$ that are parallel to the generators of the cylinder-i.e., are perpendicular to the cross section of the sample. In this case, the magnetic potential is described by two components in the cross-sectional plane and the magnetic field outside the sample is constant everywhere. Thus, due to the continuity of the tangential components of the magnetic field across the sample boundary, we also have that

$$
\mathbf{h} \times \mathbf{n}=(\boldsymbol{\nabla} \times \mathbf{A}) \times \mathbf{n}=\mathbf{H}_{e x t} \times \mathbf{n}
$$

along that surface. In addition, we apply the zero electric potential gauge $\Phi=0$. 


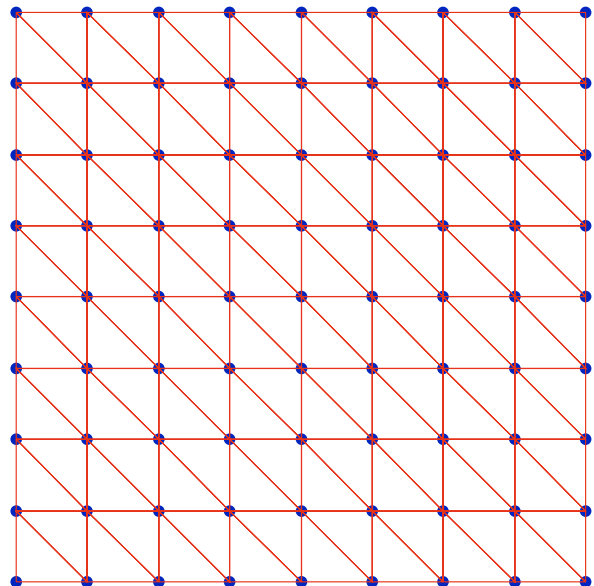

(a)

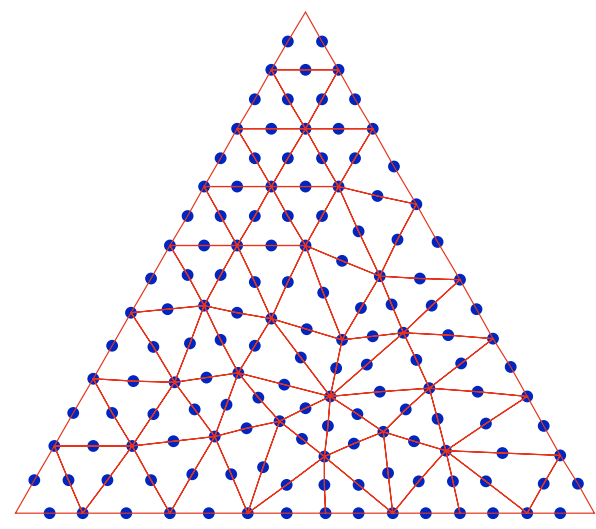

(b)

FIG. 1. (Color online) Examples of the triangulations used in the computational studies. Top: a uniform mesh in a square sample. Bottom: a conforming Delaunay triangulation in an equilateral triangle.

Characteristic scales and parameters are defined as follows: $\left|\psi_{0}\right|=(|\alpha| / \beta)^{1 / 2}$, the value of the magnitude of the order parameter that minimizes the Gibbs free energy in the absence of a field; the thermodynamic critical field strength $H_{c}=(4 \pi|\alpha|)^{1 / 2}\left|\psi_{0}\right|$, which separates the normal and superconducting states in the phase diagram for type-I superconductors; the London penetration depth $\lambda=\left(m_{s} c^{2} / 4 \pi\left|\psi_{0}\right|^{2} e_{s}^{2}\right)^{1 / 2}$ and the coherence length $\xi=\left(\hbar^{2} / 2 m_{s}|\alpha|\right)^{1 / 2}$, the length scales over which one can see appreciable changes in the value of the induced field and order parameter, respectively; and the Ginzburg-Landau parameter $\kappa=\lambda / \xi$. Dimensionless variables are defined as follows: $x \rightarrow \frac{x}{\xi}, \mathbf{H}_{e x t} \rightarrow \frac{\mathbf{H}_{e x t}}{\sqrt{2} H_{c}}, \mathbf{h} \rightarrow \frac{\mathbf{h}}{\sqrt{2} H_{c}}$, $\mathbf{j} \rightarrow \frac{2 \sqrt{2} \pi \xi}{c H_{c}} \mathbf{j}, \mathbf{A} \rightarrow \frac{\mathbf{A}}{\sqrt{2} H_{c} \xi}, \psi \rightarrow \frac{\psi}{\psi_{0}}$, and $t \rightarrow \frac{|\alpha|}{\gamma \hbar} t$. After nondimensionalization, the ratio of the relaxation time of the order parameter to that of the vector potential is $\mathcal{R} \equiv \frac{\gamma e_{s}^{2} \hbar}{\nu \beta m_{s}}$. We chose $\mathcal{R}=10 .^{27}$

The TDGL equations are discretized in time by the backward Euler method and, in space, by a Galerkin finiteelement method using continuous piecewise quadratic polynomials defined with respect to a triangulation of the sample. The resulting nonlinear discrete system is solved, at each time step, by Newton's method. Figure 1 provides examples

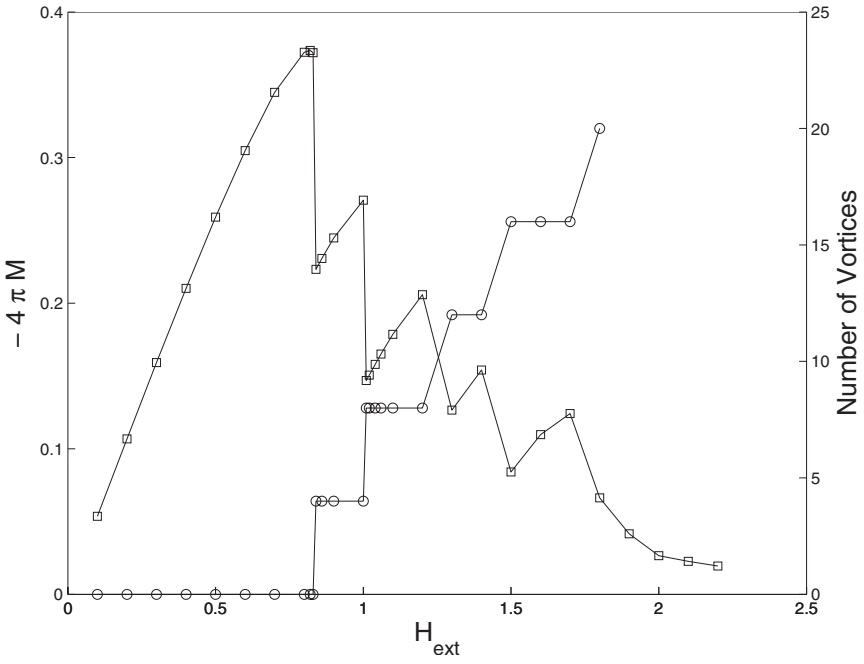

FIG. 2. The magnetization ( $\square$ ) and the corresponding number of vortices $\left({ }^{\circ}\right)$ vs the value of the external magnetic fields for a sample having square cross section of area $200 \xi^{2}$ and for $\kappa=2$.

of the triangulations used in our computational studies. On the top is a uniform mesh in a square sample, while on the bottom is a conforming Delaunay triangulation created using the TRIANGLE software package. ${ }^{28}$ The triangulations used typically contain from 300 to 1000 triangles.

\section{EFFECTS OF SAMPLE SHAPE AND SIZE ON VORTEX NUCLEATION AND CONFIGURATION}

We begin by presenting computational simulations showing how, when a mesoscopic type-II superconductor is cooled below the critical temperature and then an external magnetic field is applied, vortices nucleate at the boundary, enter into the sample, and settle into stable arrangements. This process is often referred to as zero-field cooling.

In Fig. 2, the magnetization and the corresponding number of vortices are plotted versus the value of the applied field for a cylindrical sample having a square cross section of area $200 \xi^{2}$ and for $\kappa=2$. For each value of the applied field, the simulation starts from the Meissner state and is subsequently allowed to reach a steady state. For this mesoscopic sample, jumps in the magnetization occur whenever vortices enter into the sample and the corresponding number of vortices increases by the multiple of 4 . At $H_{\text {ext }} \approx 0.84$, the Meissner state is destroyed and vortices enter the sample; see Fig. 3(a). (Note that all plots of vortex configurations display the values of $|\psi|$, with red indicating the highest values and blue the lowest. Thus, the vortex cores are found in the blue regions of the plots. In a grayscale version of the plot, the vortex cores and the superconducting region appear dark, while the intermediate region is light.) Due to the square symmetry of the sample geometry, an equal number of vortices nucleate at each side of the square. Thus, for the higher value $H_{\text {ext }} \approx 1.01$, two vortices enter from each side, resulting in eight vortices in the sample [see Fig. 3(b)] and so on. Note that for $H_{\text {ext }}>1.8$, the vortices become so closely packed that they become very difficult to count. 


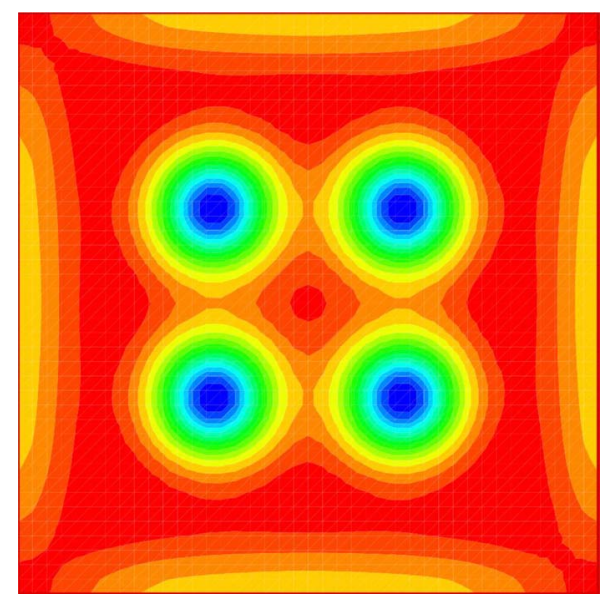

(a)

$\mathrm{H}_{\mathrm{ext}}=0.84$

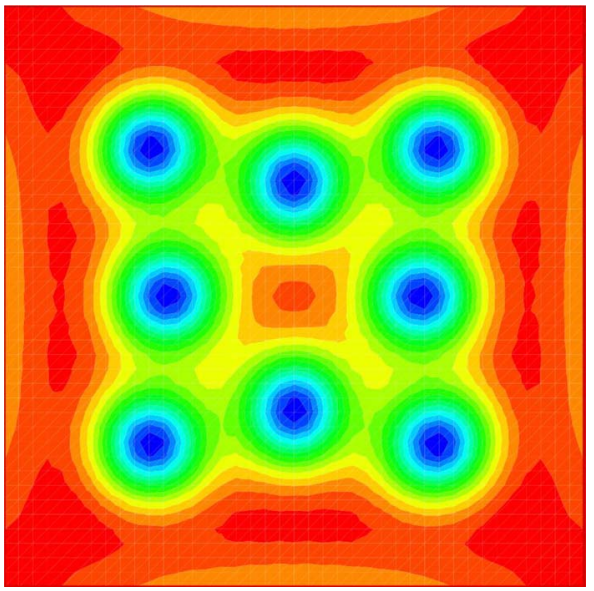

(b)

$$
\mathrm{H}_{\mathrm{ext}}=1.01
$$

Figures 3(c) and 3(d) show the steady-state vortex configurations for an equilateral triangle having the same areai.e., $200 \xi^{2}$. The same trend occurs as for the square sample; i.e., the same number of vortices nucleate at each side and, as the applied magnetic field increases, more vortices enter each side simultaneously. Thus, if a cylindrical sample has a perfectly symmetric cross section, only configurations consistent with the sample symmetry can appear under a uniform magnetic field. This allows vortices to nucleate and enter the sample only in multiples of the number of sides-e.g., multiples of 4 in squares and 3 in triangles.

For the square, there are four vortices for $H_{\text {ext }}=0.84$ and 8 for $H_{\text {ext }}=1.01$, as shown in Figs. 3(a) and 3(b). For an equilateral triangle of the same area, there are three vortices for $H_{e x t}=0.84$ and 6 for $H_{e x t}=0.96$. For the latter case, two vortices enter the sample at each side.

Although we did not conduct as many simulations for equilateral triangles as for squares, we have found that for triangles, $H_{\text {ext }}=0.84$ is the magnetic field for the first vortex entry and $H_{\text {ext }}=0.96$ is the magnetic field for which the transition from the three-vortex configuration to six-vortex configuration occurs. The corresponding values for the square are $H_{e x t}=0.84$ and $H_{e x t}=1.01$. Since the sides of the equilateral triangle are longer than the sides of the square, this indicates that it is easier for vortices to enter along longer surfaces.
FIG. 3. (Color online) Steadystate vortex configurations for cylindrical samples with symmetric cross sections of area $200 \xi^{2}$ and for $\kappa=2$.
This trend was examined more closely by considering samples with rectangular cross sections. Figure 4(a) shows the steady-state vortex configuration for a rectangle of aspect ratio 2 and having the same area as the square of Fig. 3. For the rectangular sample, two vortices entered the system through each of the two longer sides and none through the shorter sides. The shorter sides of the rectangle are "choked" for vortex penetration. On the other hand, adding a concavity on one of the shorter sides as in Fig. 4(b), while keeping the same area, allows for the nucleation of an additional vortex at the concavity, resulting in a total of five vortices in the steady-state configuration. For both cases shown in Fig. 4 we have set $\kappa=2$ and $H_{\text {ext }}=1.0$.

Additional simulations for rectangles with other aspect ratios and for isosceles triangles with and without concave indentations indicate that there is a competition between the longer sides and concave surfaces. However, it is clear that concavity provides a localized area where the surface barrier is lower than in its neighborhood which could prove very useful for technological applications.

Enomoto and Okada ${ }^{18}$ examined the effect of surface irregularities on the surface barrier by randomly placing surface defects of the size of the London penetration depth $\lambda$ along the sample boundary. We also examine the effect of a surface defect, but we choose a single defect in the form of a sliver-shaped indentation at one location on the boundary. 


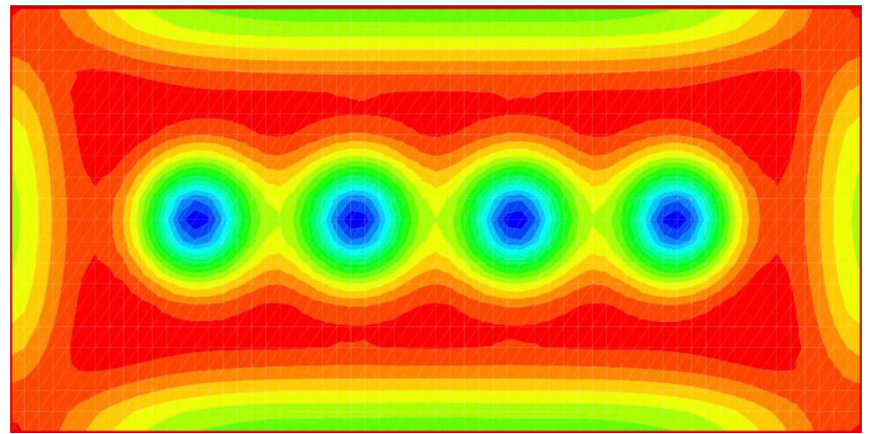

(a)

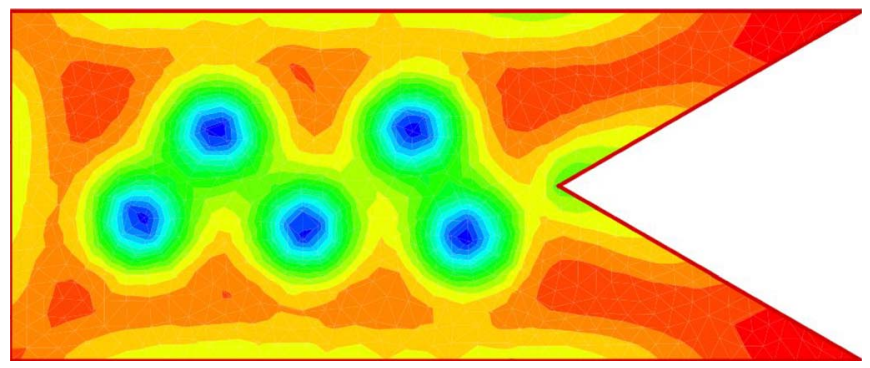

(b)

FIG. 4. (Color online) Steady-state vortex configurations in a rectangle (a) and a rectangular sample with the same area but having a concave indentation (b); $H_{\mathrm{ext}}=1.0$ and $\kappa=2$.

We then study how such a small perturbation in the sample geometry affects the vortex states. Figure 5 illustrates the types of meshes we use for both indented and unindented samples; these meshes were created using the TRIANGLE software package.

The thin sliver (concave surface) is placed at the middle of the bottom side of a square sample having area $346 \xi^{2}$ and $\kappa=4$ that is subject to a magnetic field $H_{e x t}=0.84$. Figure 6 shows time snapshots of the vortex nucleation and penetration process. Starting from the Meissner state, magnetic fields attempt to penetrate from all four sides of the square. However, vortices nucleate only at the tip of the sliver and enter the system one by one. Note that without the indentation the system has four vortices. ${ }^{13}$ The curvature of the vector curves of the shielding current is the largest around the tip of the indentation, resulting in the most severe suppression of the value of the order parameter ${ }^{21,29}$ and the nucleation of a vortex at that location. Due to the small, mesoscopic size of the sample, for this value of the applied field, vortices do not nucleate at the sides of the square sample when the indentation is present. Note that there is a tendency for the vortices to conform, as well as they can, to the symmetry of the sample (which is now limited to reflections about the vertical midline), even at the time $t=4491.5$ as well as at the steady state.

For Fig. 7, we have the same setting as that for Fig. 6, except that now the applied field has the higher value $H_{\text {ext }}$ $=0.9$. While the number of vortices entering the sample is the same as for $H_{\text {ext }}=0.84$, now one vortex enters $($ at $t \approx 18.9$ ) from the top side.

The angle between the two surfaces of the slender indentation is small so that the surfaces can be regarded as being

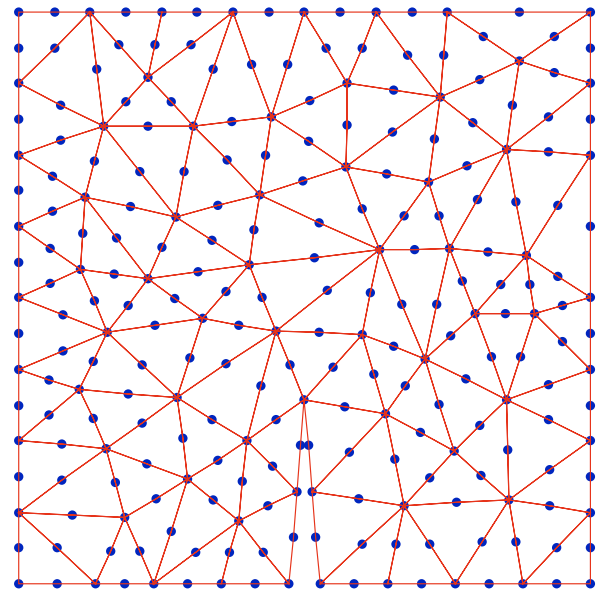

(a)

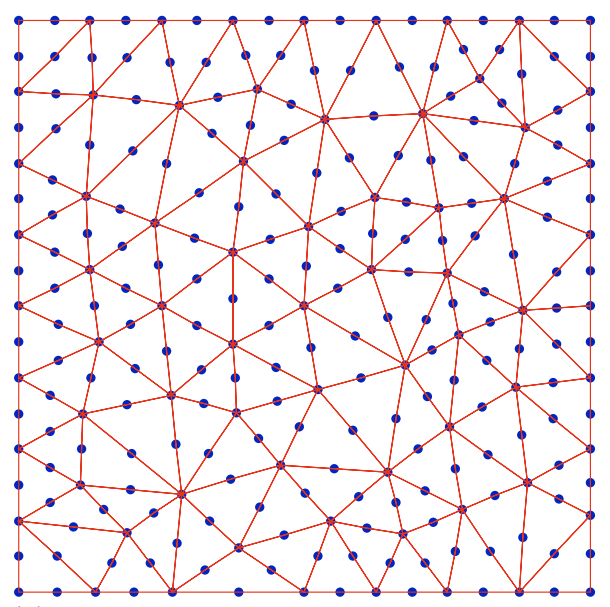

(b)

FIG. 5. (Color online) Examples of the triangulations used for dented and unindented samples.

nearly parallel. Thus, the currents along the surfaces of the slender indentation flow in nearly opposite directions and, hence, induce negligible magnetic fields. Therefore, the overall shielding current profile for the indented sample does not differ much from that for an unindented sample, and, in particular, away from the indentation, the shielding currents along the surface of the sample are not appreciably affected by the indentation. However, since the "extra" current due to the indentation increases the total energy of the system, the overall level of the shielding current that repel vortices ${ }^{15}$ should be slightly lower for an indented sample. This means that the surface barrier is not as strong, making it easier for vortices to enter the sample along the sides as well as from the tip of the indentation.

The resultant vortex dynamics is seen to involve strong magnetic interactions and is influenced by the magnetic fields at all sample boundary surfaces. In a previous study ${ }^{21}$ of a bulk superconductor with a single surface defect, the variation of the first-vortex-entry field was attributed solely to the size of the defect; i.e., the first-vortex-entry field decreases as the length (depth into the sample) of defect increases and increases as the width of defect increases. However, in our study for finite samples of mesoscopic size, there are always strong magnetic interactions between different 

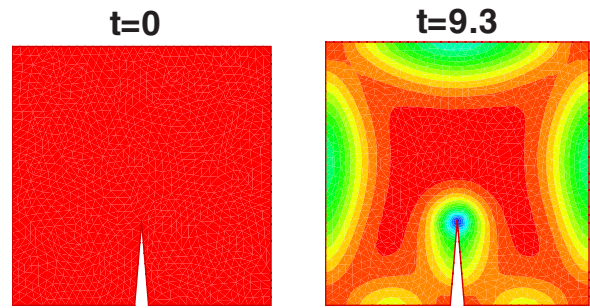

$t=111.7$
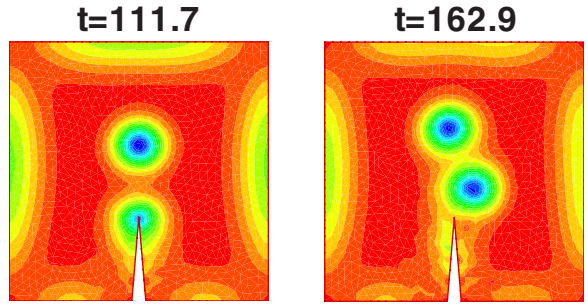

$t=201.3$
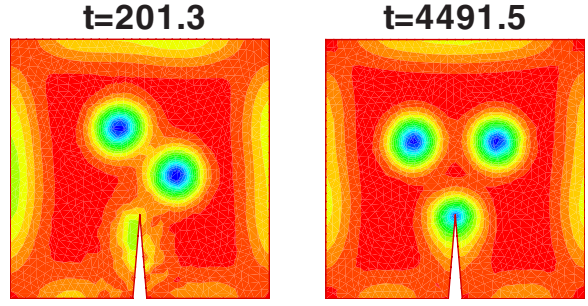

$t=4585.25$
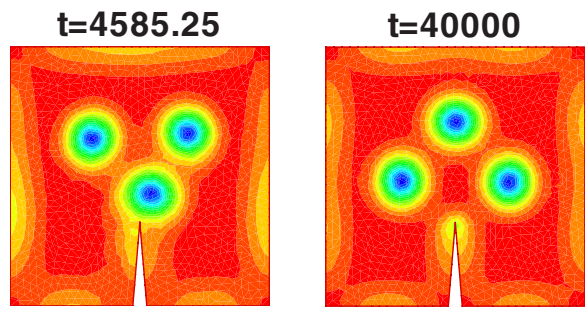

FIG. 6. (Color online) Time history of the vortex nucleation and penetration process for an indented sample; the area is $346 \xi^{2}$, $H_{\text {ext }}=0.84$, and $\kappa=4$.

boundary surfaces of the sample; e.g., the magnetic field on one side tends to repel the magnetic fields coming from the other sides.

However, this effect is reduced as the sample size increases. Figure 8 shows the penetration process in a sample having area $1024 \xi^{2}$. At an early stage, a single vortex nucleates at the tip of the indentation and, due to their longer length, each of the three unindented sides allows two vortices to simultaneously nucleate. No vortices nucleate along the side of the square along which the indentation is located. Once these seven vortices have entered the system, any additional vortices attempting to nucleate anywhere on the boundary should experience a higher surface barrier due to the repulsion force exerted by the vortices already inside the sample. ${ }^{15,20}$ In fact, only the tip of the indentation has a low enough surface barrier to allow for further vortex nucleation. Thus, at later stages, no more vortices nucleate along the unindented sides, but seven more vortices do enter the sample at the tip of the indentation.

Since vortices can be made to enter one by one by using indentations of the type discussed above, it should be pos-
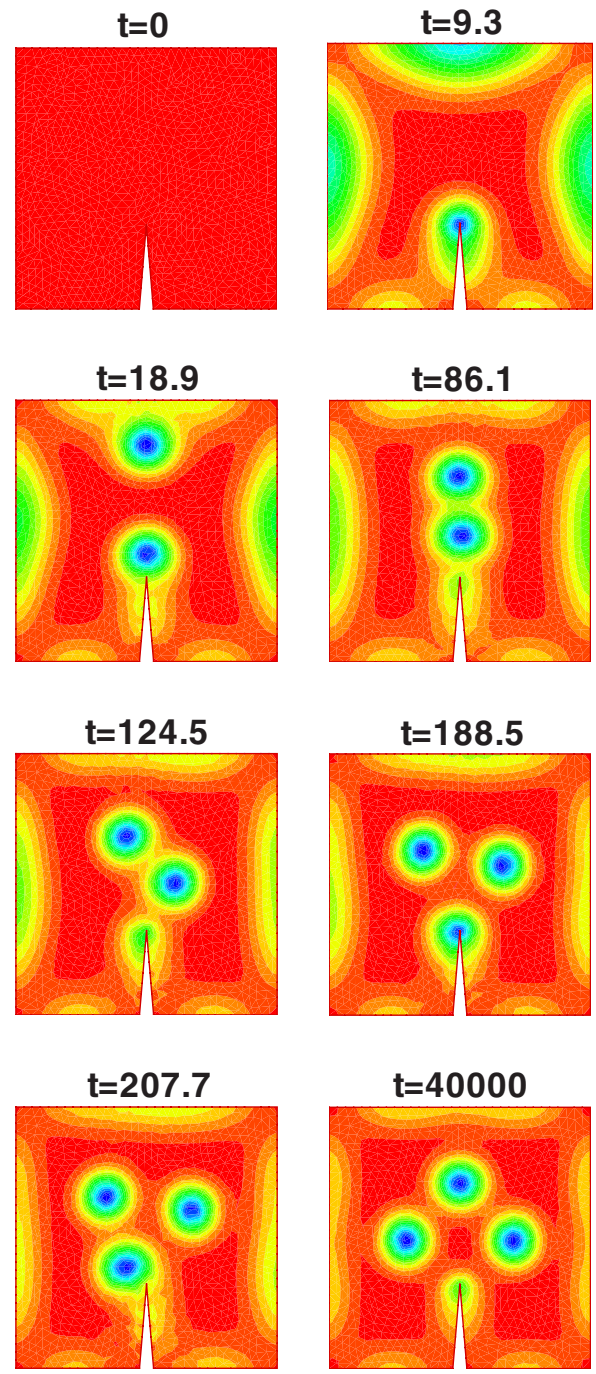

FIG. 7. (Color online) Time history of the vortex nucleation and penetration process for an indented sample; the area is $346 \xi^{2}$, $H_{\text {ext }}=0.9$, and $\kappa=4$.

sible to obtain a specified number and arrangement of vortices in a sample through careful control of the magnetization process. Thus, our method provides a good potential for nanoscience applications. For example, a controlled local magnetic field (vortices) in a domain of submicron scale (only tens of coherence lengths) can provide resolutions that may surpass those of existing high-precision devices such as scanning superconducting quantum interference device (SQUID) microscopes. ${ }^{30}$

Experimental studies of superconducting and normal phase boundaries are carried out by electrical transport measurements on mesoscopic samples. Wedge-shaped electrical contact leads are commonly used to minimize their influence on the superconducting properties of the sample. However, starting from the normal state in a nonzero magnetic field, as the temperature decreases the contacts become superconducting first and induce the nucleation of superconductivity in the sample. Thus, the shape of the resistive transition is quite sensitive to the presence of contacts and displays doubletransition points. ${ }^{31}$ This is somewhat a reversed physical picture (normal to superconducting transition) compared to our 

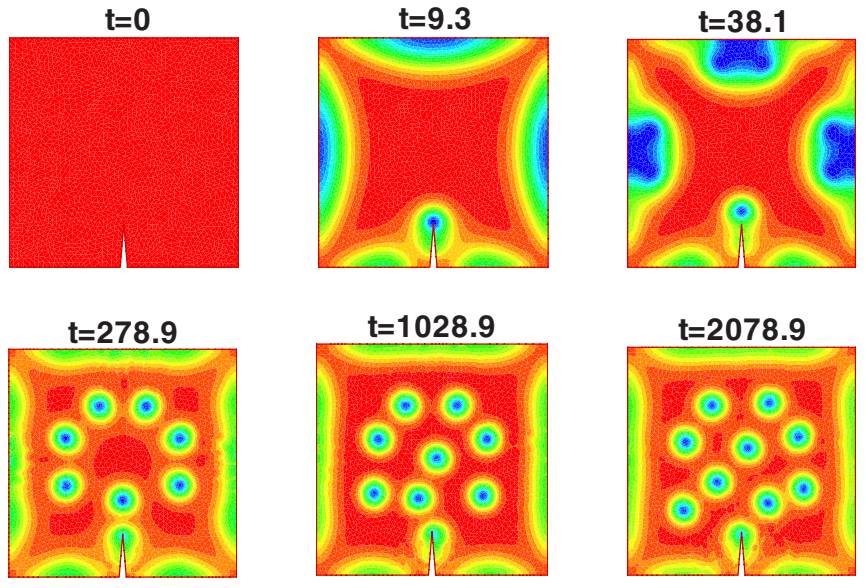

$t=4928.9$
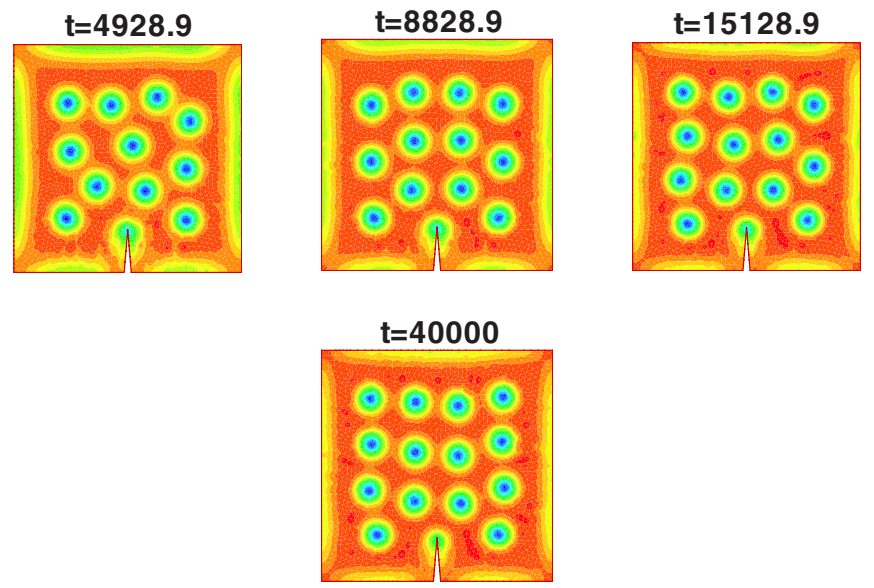

FIG. 8. (Color online) The vortex nucleation and penetration process in a sample of area $1024 \xi^{2}$ for $H_{\text {ext }}=0.84$ and $\kappa=4$.

study for which vortices penetrate the samples in the Meissner state. However, it is clear that the "indentation method" may also be used for the computational modeling of such situations.

\section{UTILIZING SURFACE DEFECTS FOR DETERMINING EQUILIBRIUM STATES}

Our simulations, such as those depicted in, e.g., Fig. 3, result in steady-state arrangements of vortices that conform to the symmetry of the samples. Round-off error alone is not sufficient to break symmetry, even over the long times over which one sometimes has to integrate. Steady-state vortex configurations that conform to sample symmetries do not necessarily correspond to (global) minimizers of the Gibbs free energy. It may not even have the same number of vortices as does the configuration that minimizes that energy. In real situations, symmetry is broken in two ways. First, one has imperfections in the data; e.g., the sample surface invariably is slightly imperfect or even the applied field is not perfectly uniform. Second, thermal fluctuations introduce random, and therefore nonsymmetric, perturbations into the system. The TDGL model we use does not account for the effects of thermal fluctuations so that our results correspond to samples at a low enough temperature so that thermal fluc- tuations are not able to overcome any energy barrier that prevents escape from a configuration that corresponds to a local minimizer of the energy.

It is well known that the configurational space of superconducting states has (possibly infinitely) many local minimizers of the Gibbs free energy. The inherent tendency of vortices to configure themselves into a triangular lattice in a bulk sample competes with the effects due to boundaries. For samples of small enough size, the geometry wins this battle. The surface barrier can trap the system in a metastable state with a vortex configuration consistent with the symmetry of the sample without allowing for the entry or exit of additional vortices.

Determining equilibrium states having minimum Gibbs free energies through computational simulations is a challenging task since one often needs to escape from a local minimizer. To determine the equilibrium state, one needs to make sure the system has sufficient energy to overcome local energy barriers in configurational space. For example, a Langevin force term can be added to the TDGL equation to model the effects of thermal fluctuation. ${ }^{32-34}$ This term breaks the symmetry of the computational model by energizing the system to jump out of the local minimum in the energy and over the energy barrier. ${ }^{3}$ However, this approach increases the computing time greatly. (This method is essentially the same as "simulated annealing."32,35) Furthermore, we believe that this approach is not practical since it is likely to be difficult to determine the appropriate rate of cooling and the starting temperature.

In a previous study, ${ }^{13}$ a controlled number of vortices, located at random positions, were "seeded" into a sample and then allowed to evolve to a steady-state configuration. The Gibbs free energy of each of the states so obtained were compared to determine what number of vortices yielded the minimum energy configuration. This approach, which seeks the equilibrium state among possible vortex configurations already inside the sample, does not have to deal directly with surface barrier, unlike the approach we use.

We now examine the possibility of finding equilibrium states by using the property that vortices can be made to enter a sample one at a time at the tip of a thin indentation of the boundary. The presence of the thin indentation lowers the surface barrier and, when placed at an asymmetric position, can also be used to break the symmetry. Once a steady-state configuration is attained, the indentation is removed and the vortices are allowed to further evolve to a steady-state configuration for an unindented sample-i.e., for a symmetric sample. We then compare the Gibbs free energies of configurations having different numbers of vortices to determine the one that minimizes the energy.

As was shown in Sec. III (see Figs. 6-8), the surface barrier at smooth surfaces can be as low as that at indentation, unless the magnetic field is sufficiently low. Thus, it is difficult to confine the vortex nucleation and penetration process to just the indentation. Since vortices simultaneously enter from all the smooth sides of the boundary, this situation makes it difficult to produce configurations with an arbitrary specified number of vortices. However, we do know that, at a fixed value of the applied magnetic field, vortices entering the sample from the boundary add to the repulsion prevent- 

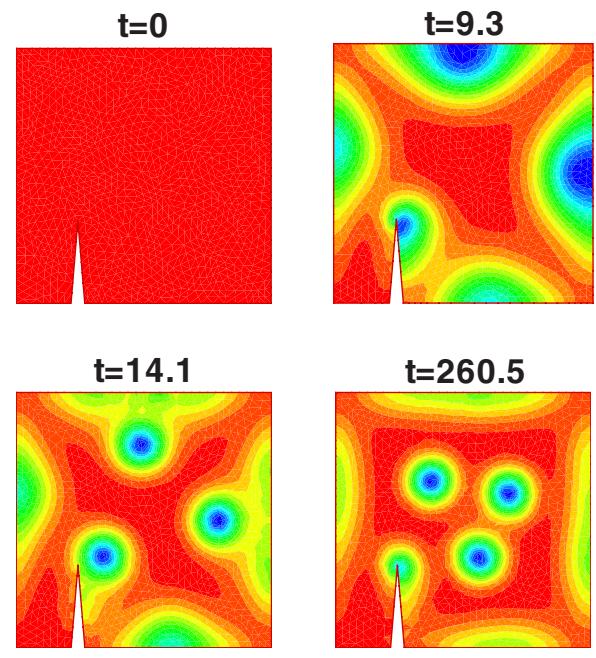

$\mathrm{t}=\mathbf{2 7 9 . 7}$

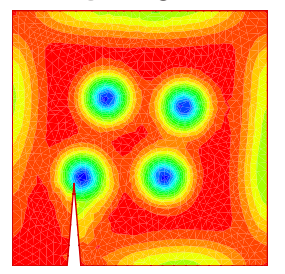

FIG. 9. (Color online) Vortex nucleation and penetration for an indented square sample having area $346 \xi^{2}$ and $\kappa=4$ for $H_{\text {ext }}=1.0$. The location of indentation is chosen to break symmetry.

ing additional vortices from entering. We believe that by raising the applied magnetic field from zero by small increments, the vortex penetration process can be controlled to occur only at the indentation until a specified number of vortices have entered the sample. We leave this approach for future study.

Instead, here we simply create, for several different $H_{\text {ext }}$, steady-state vortex configurations in a sample with square cross section which has a thin indentation in the bottom side. Since the area removed by the indentation is a small fraction of the total area of the square, for the same number of vortices the magnetic fluxes inside the samples with and without the indentation are close to each other. We use the steadystate configurations for the indented sample as initial conditions for determining the vortex dynamics in the unindented sample. All of these are carried out using a single value of the applied field-i.e., the value for which we are trying to determine the equilibrium configuration. The steady-state configurations obtained for the latter case will, most of the time, conserve the number of vortices due to surface barrier. By comparing the Gibbs free energies of the steady states so obtained that have a different number of vortices, we can determine which state-state configuration has lowest energy; we identify that configuration with the equilibrium state. ${ }^{36}$

We now illustrate how this approach is used to obtain steady-state vortex configurations containing different numbers of vortices, $n_{\infty}$, and in particular, configurations that do not conform to the symmetry of a sample. For Fig. 9, we have a square sample of area $346 \xi^{2}$ with a small indentation asymmetrically placed along its bottom side. We also have that $\kappa=4$ and $H_{e x t}=1.0$. Vortices enter the sample from both the tip of the indentation and from the sides of the square.

By varying the value of the applied field, vortex configurations having different values of $n_{\infty}$ are created. Figure 10 presents the results of nine cases, labeled (a) through (i). For each case, two figures are shown, presenting the steady-state vortex configuration in the indented sample (left, called "left figure" henceforth) and the nonindented square (right, called "right figure" henceforth). The left figures of Fig. 10 show the steady-state configurations for the indented sample obtained for the applied field values $H_{e x t}=1.0,1.05,1.10$, $1.144,1.357,1.429,1.454,1.593$, and 1.732 , respectively. These values were rather arbitrarily chosen to obtain the desired number of vortices. Note that these configurations contain 4-12 vortices plus an additional vortex at the tip of the indentation.

We next remove the indentation, set the applied field to $H_{e x t}=1.144$, and let each of the nine steady-state configurations displayed in the left figure of Fig. 10 evolve to new steady-state configurations which are shown in the right figure of Fig. 10. We emphasize that the configurations shown in the left figures of Fig. 10 were determined using different values for the applied field; after the indentation is removed, the same applied field value is used for all configurations. The vortex formed around the tip of the indentation remains inside the sample in cases (a)-(f), but is expelled from the sample for cases (g)-(i). The resulting configurations in the right figures of Fig. 10 have different numbers of vortices, with the exception of (f) and (g), both of which not only have ten vortices each, but, although having evolved from different initial states, have vortex configurations which are merely rotated versions of each other. Also, one observes in the right figures of rows $(\mathrm{g})$ and $(\mathrm{h})$ the competition between the desire of the vortices to arrange themselves in the Abrikosov lattice and to conform with the sample boundary.

The Gibbs free energies $G$ corresponding to each of configurations in the right figure of Fig. 10 are plotted, in Fig. 11 , versus the number of vortices present in the configurations. From that plot, we see that the configuration having the smallest Gibbs free energy (e) is the one with nine vortices. Thus, we conclude that for the square sample of area $346 \xi^{2}$ and for $\kappa=4$ and for $H_{e x t}=1.144$, the equilibrium configuration contains nine vortices and is given by the figure in the right figure of Fig. 10(e). Note that the nine vortices in that configuration are symmetrically arranged and that, if one started a simulation for $H_{e x t}=1.144$ from the Meissner state, one would obtain a steady-state configuration having only eight vortices.

Another example is provided in Fig. 12. On the top is the steady-state configuration for an indented square of area $346 \xi^{2}$ with $H_{e x t}=1.0$ and $\kappa=4$. But now that result is used as an initial condition for a simulation for an unindented region with $H_{e x t}=0.84$. The steady-state configuration for the latter case is shown on the bottom of Fig. 12. This configuration is identical with the equilibrium state found in a previous study ${ }^{13}$ for a square of the same area, applied field, and $\kappa$.

\section{SUMMARY AND CONCLUSION}

Steady-state configurations and transient behaviors of magnetic vortices for various values of the applied magnetic 


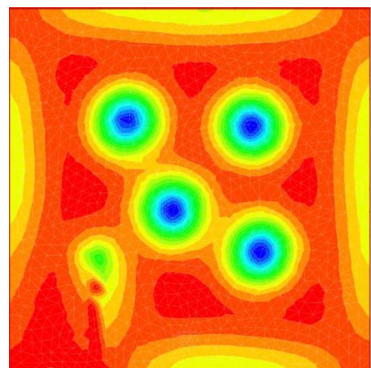

(a)

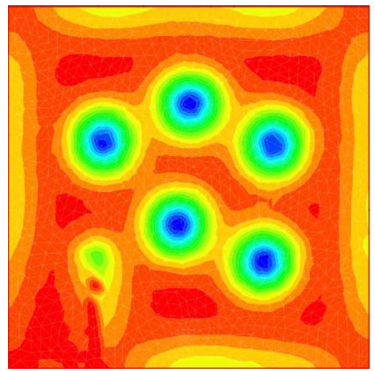

(b)

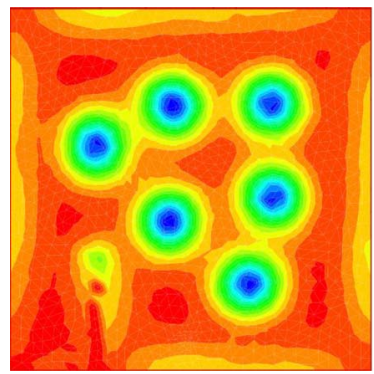

(c)

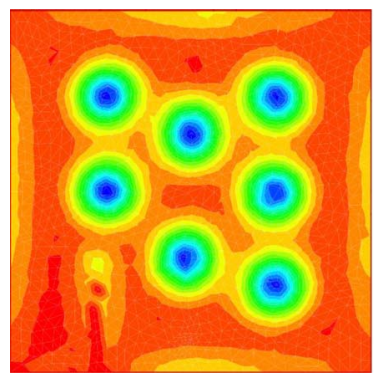

(d)

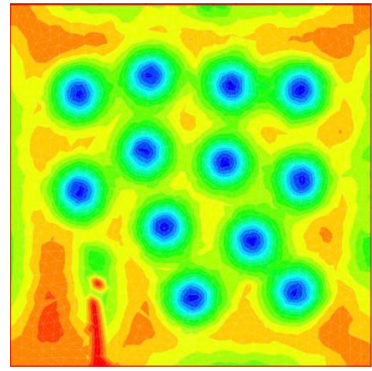

(i)
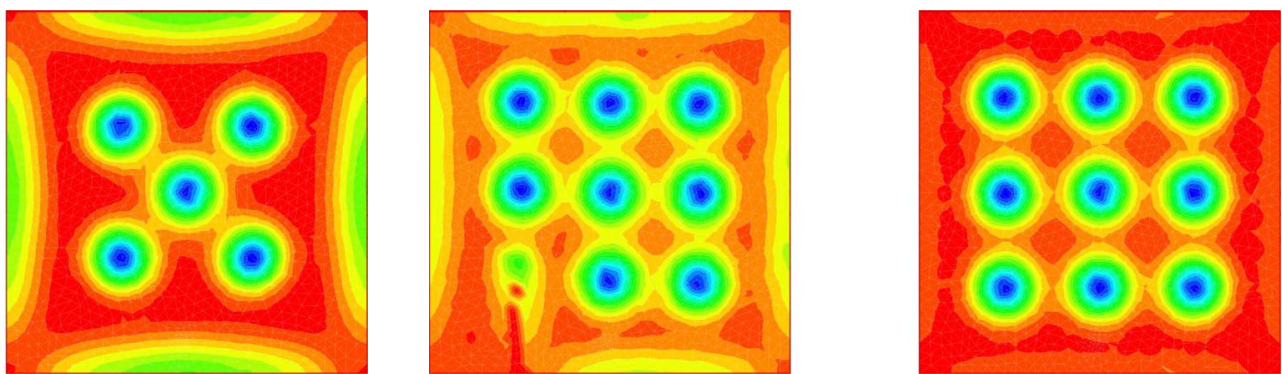

(e)
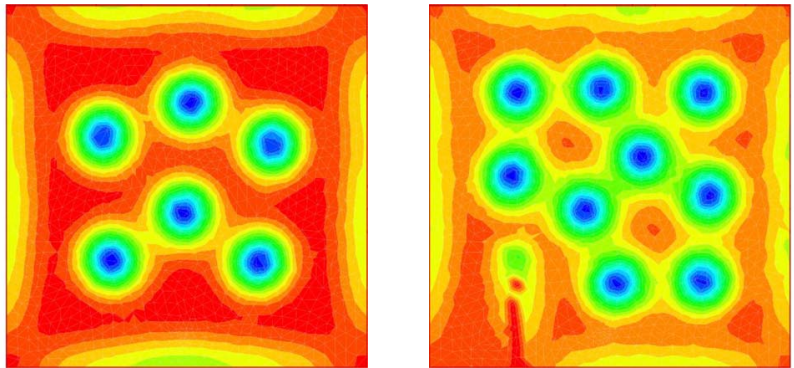

(f)
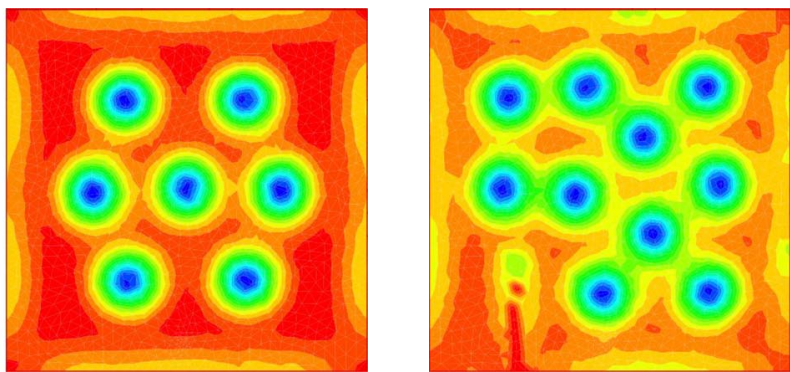

(g)
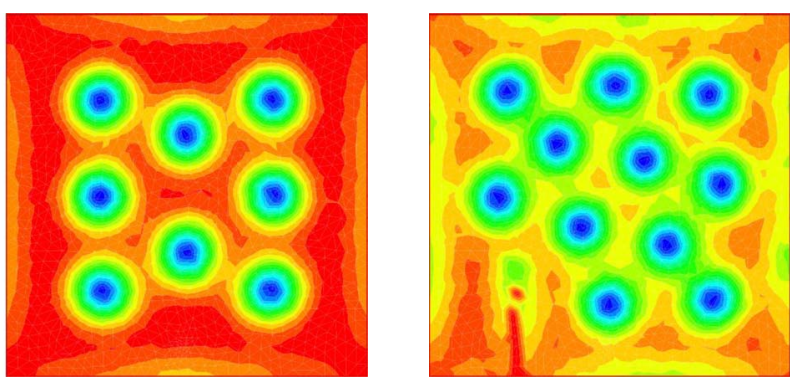

(h)

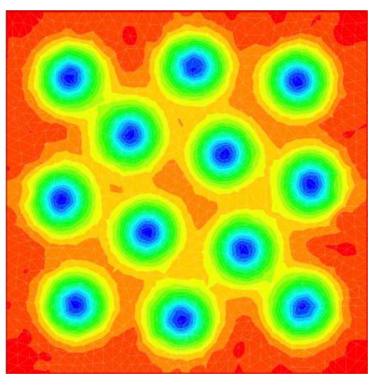

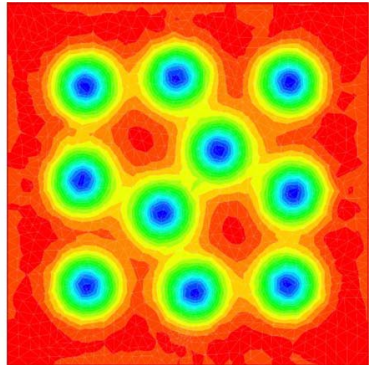
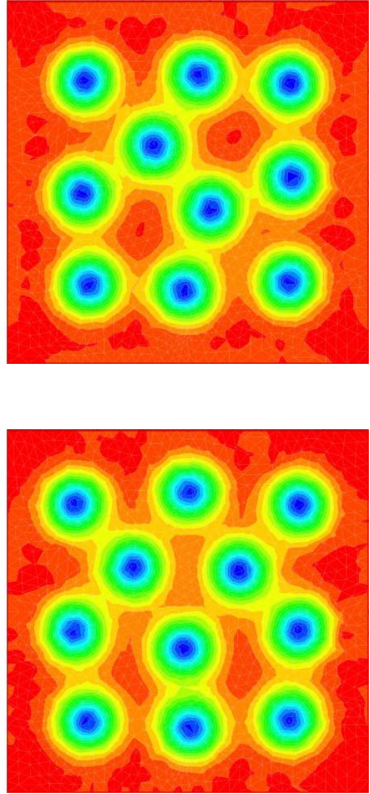

FIG. 10. (Color online) Steady-state vortex configurations for a square sample of area $346 \xi^{2}$ with $\kappa=4$. Left figures: configurations for an indented sample obtained using different values for the applied field. Right figures: configurations for an unindented sample obtained from the corresponding configurations in the left column using the applied field value $H_{\text {ext }}=1.144$. 


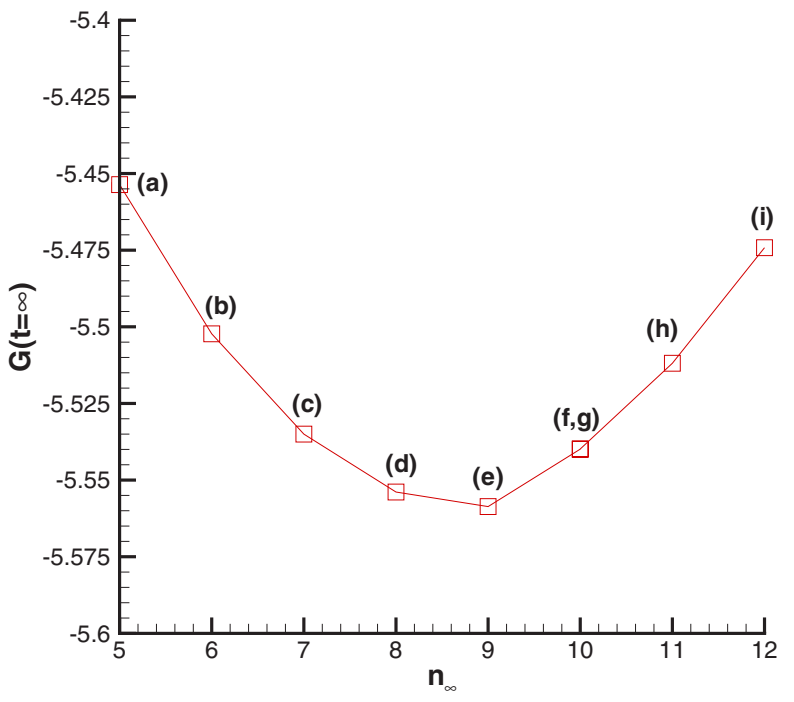

FIG. 11. (Color online) The steady-state total Gibbs free energies $G$ of the steady-state configurations in the right figure of Fig. 10 vs the number of vortices $n_{\infty}$, in the configurations.

field $H_{\text {ext }}$ have been presented. The effect of sample dimension and shape on the surface barrier was examined. When we start the solution with a uniformly superconducting initial condition and the sample has perfect square or triangular symmetry, both the number of vortices and their steady-state configurations are governed by the sample geometry. In particular, vortices enter the samples in multiples of the symmetry number. More vortices enter as the sample dimension grows, and when a small indentation is made on the sample boundary, vortices enter the system one by one from the tip of the indentation.

Equilibrium states in actual samples may not have such symmetric configurations due to symmetry-breaking surface defects that form vortex-nucleation centers or when thermal fluctuations are sufficiently strong to move the system out of metastable states but are not too strong to melt the vortex lattice. One could have determined these true equilibrium states by adding additional terms in the equations to simulate thermal fluctuations, but here, we have devised a different approach which we believe is more efficient. Since thin indentations lower the surface barrier and also break the symmetry when placed at an asymmetric position, the resulting steady-state configuration is expected to be close to a steadystate configuration of an unindented sample. (This follows because the area removed by the indentation is a small fraction of the whole area and the magnetic fluxes inside the systems with and without the indentation are close to each other, for the same number of vortices.) Thus, further relaxation from this state after removing the indentation will find a steady-state configuration for the symmetric sample. Since the surface barrier at smooth surfaces can be as low as that at indentation (unless the magnetic field is sufficiently low), vortices have also entered from the boundary surfaces and add to the repulsion preventing additional vortices from entering at a fixed magnetic field. The number of vortices in the symmetric sample is, for the most part, kept the same as for the indented samples due to surface barrier. In this manner, we can create steady-state vortex configurations in a square

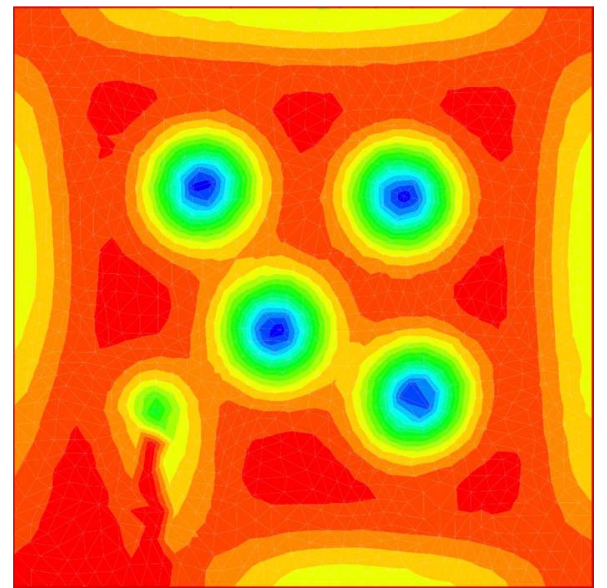

(a)

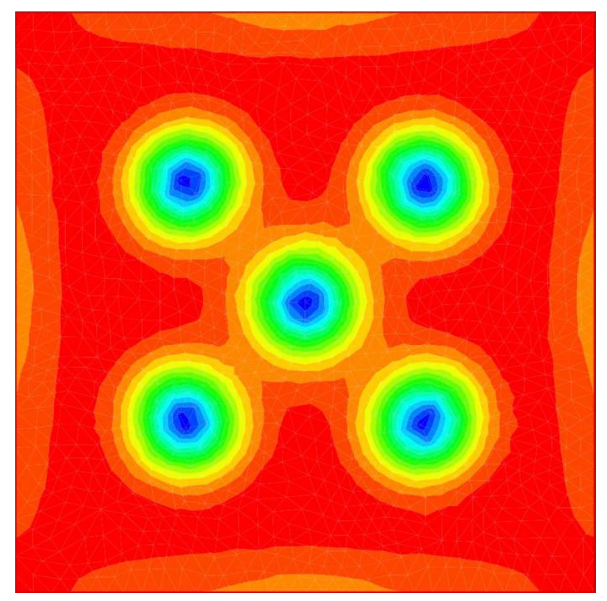

(b)

FIG. 12. (Color online) Top: initial configuration determined using an indented sample and $H_{\text {ext }}=1.0$. Bottom: equilibrium configuration for the unindented sample for $H_{\text {ext }}=0.84$.

sample with differing number of vortices for the same value of the applied field. By comparing the Gibbs free energies of these steady-state vortex configurations, we can determine which one has the lowest Gibbs free energy. We identify that configuration as the equilibrium state for the given value of the applied field and, in this way, we determine the number of vortices present in the equilibrium configuration.

Our indentation method is an alternative to the vortex "seeding" method ${ }^{13}$ (for which vortices are artificially introduced into a sample at random locations) for finding equilibrium configurations. We believe that, by raising the external magnetic field from zero using small increments, the vortex penetration process can be controlled to occur only at the indentation until the desired number of vortices have entered. In this way, we can obtain a vortex state very close to the equilibrium state in a square without an indentation. We intend to pursue this approach in a future study.

\section{ACKNOWLEDGMENT}

C.-R.H. acknowledges some support in the summer of 2006 from the Texas Center for Superconductivity at the University of Houston. 
${ }^{1}$ V. L. Ginzburg and L. D. Landau, Zh. Eksp. Teor. Fiz. 20, 1064 (1950).

${ }^{2}$ P. G. de Gennes, Superconductivity in Metals and Alloys (Addison-Wesley, Reading, MA, 1989).

${ }^{3}$ M. Tinkham, Introduction to Superconductivity (McGraw-Hill, New York, 1996).

${ }^{4}$ Q. Du, M. D. Gunzburger, and J. S. Peterson, SIAM Rev. 34, 54 (1992).

${ }^{5}$ Q. Du, M. D. Gunzburger, and J. S. Peterson, Phys. Rev. B 51, 16194 (1995).

${ }^{6}$ B. J. Baelus and F. M. Peeters, Phys. Rev. B 65, 104515 (2002).

${ }^{7}$ V. A. Schweigert and F. M. Peeters, Phys. Rev. B 57, 13817 (1998).

${ }^{8}$ V. A. Schweigert, F. M. Peeters, and P. Singha Deo, Phys. Rev. Lett. 81, 2783 (1998).

${ }^{9}$ P. Singha Deo, V. A. Schweigert, F. M. Peeters, and A. K. Geim, Phys. Rev. Lett. 79, 4653 (1997).

${ }^{10}$ J. Bonca and V. V. Kabanov, Phys. Rev. B 65, 012509 (2001).

${ }^{11}$ V. R. Misko, V. M. Fomin, J. T. Devereese, and V. V. Moshchalkov, Physica C 369, 361 (2002).

${ }^{12}$ V. R. Misko, V. M. Fomin, J. T. Devreese, and V. V. Moshchalkov, Phys. Rev. Lett. 90, 147003 (2003).

${ }^{13}$ S. Kim, C.-R. Hu, and M. J. Andrews, Phys. Rev. B 69, 094521 (2004).

${ }^{14}$ A. A. Abrikosov, Zh. Eksp. Teor. Fiz. 32, 1442 (1957) [Sov. Phys. JETP 5, 1174 (1957)].

${ }^{15}$ C. P. Bean and J. D. Livingston, Phys. Rev. Lett. 12, 14 (1964).

${ }^{16}$ P. G. de Gennes, Solid State Commun. 3, 127 (1965)

${ }^{17}$ H. J. Fink and A. G. Presson, Phys. Rev. 182, 498 (1969).

${ }^{18}$ Y. Enomoto and K. Okada, J. Phys.: Condens. Matter 9, 10203 (1997).

${ }^{19}$ A. S. Mel'nikov, I. M. Nefedov, D. A. Ryzhov, I. A. Shereshevskii, V. M. Vinokur, and P. P. Vysheslavtsev, Phys. Rev. B 65, 140503(R) (2002).

${ }^{20}$ A. D. Hernández and D. Domínguez, Phys. Rev. B 65, 144529 (2002).

${ }^{21}$ D. Y. Vodolazov, Phys. Rev. B 62, 8691 (2000).

${ }^{22}$ L. Burlachkov, M. Konczykowski, Y. Yeshurun, and F. Holtzberg, J. Appl. Phys. 70, 5759 (1991).

${ }^{23}$ F. Bass, V. D. Freilikher, B. Ya. Shapiro, and M. Shvaster, Physica C 260, 231 (1996).

${ }^{24}$ A. Yu. Aladyshkin, A. S. Mel'nikov, I. A. Shereshevsky, and I. D. Tokman, Physica C 361, 67 (2001).

${ }^{25}$ A. Buzdin and M. Daumens, Physica C 294, 257 (1998).

${ }^{26}$ L. P. Gor'kov and N. B. Kopnin, Sov. Phys. Usp. 18, 496 (1976).

${ }^{27}$ According to microscopic theories $\mathcal{R}$ would be either 5.79 in the case of strong electron-phonon relaxation [R. J. Watts-Tobin, Y. Krähenbühl, and L. Kramer, J. Low Temp. Phys. 42, 459 (1981)] or 12 for superconductors with a high concentration of paramagnetic impurities \{L. P. Gor'kov and G. M. Éliashberg, Zh. Eksp. Teor. Fiz. 54, 612 (1968) [Sov. Phys. JETP 27, 328 (1968)]\}. See also Ref. 19 and R. S. Thompson and C.-R. Hu, Phys. Rev. Lett. 27, 1352 (1971). Note that there is only one relaxation time in the simplified TDGL equation considered in Ref. 19, whereas there are two relaxations times in the TDGL equation solved here.

${ }^{28}$ Jonathan Richard Shewchuk, in Applied Computational Geometry: Towards Geometric Engineering, edited by Ming C. Lin and Dinesh Manocha, Volume 1148 of Lecture Notes in Computer Science (Springer-Verlag, Berlin, 1996), pp. 203-222.

${ }^{29}$ A. C. Rose-Innes and E. H. Rhoderick, Introduction to Superconductivity (Pergamon Press, New York, 1978).

${ }^{30}$ S. Kim, C.-R. Hu, and M. J. Andrews, Phys. Rev. B 74, 214511 (2006).

${ }^{31}$ M. Morelle, G. Teniers, L. F. Chibotaru, A. Ceulemans, and V. V. Moshchalkov, Physica C 369, 351 (2002); M. Morelle, Y. Bruynseraede, and V. V. Moshchalkov, Phys. Status Solidi B 237, 365 (2003).

${ }^{32}$ M. M. Doria, J. E. Gubernatis, and D. Rainer, Phys. Rev. B 41, 6335 (1990).

${ }^{33}$ R. Kato, Y. Enomoto, and S. Maekawa, Phys. Rev. B 47, 8016 (1993).

${ }^{34}$ J. Deang, Q. Du, and M. D. Gunzburger, Phys. Rev. B 64, 052506 (2001).

${ }^{35}$ W. H. Press, B. P. Flannery, S. A. Teukolsky, and W. T. Vetterling, Numerical Recipes (Cambridge University Press, Cambridge, England, 1989), Sec. 10.9.

${ }^{36}$ Mel'nikov et al. (Ref. 19) used a small defect (normal metal) on the boundary of the samples to disturb the fourfold symmetry of their numerical scheme to obtain the vortex states with odd total vorticity. With our deep-cut defect, we can more likely find the lowest-energy vortex configuration at each applied field and vorticity, since a small surface defect cannot change much of the local surface magnetic pressure, which is the driving force for vortex entry, and only a highly symmetric steady state can be affected drastically by the symmetry-breaking small surface defect. Note also that in our paper, sample defects were used merely as a tool to more quickly find the true equilibrium states of the perfectly symmetric samples with no defects. This trick allows the true equilibrium solutions that do not have the symmetry of the sample to be more easily obtained. In addition, Mel'nikov et al., used larger defects to examine the stability of a maximally symmetric vortex configuration near the critical field $H_{c 3}$. However, we have considered applied fields substantially below $H_{c 2}$, so we found no antivortices or multiply quantized vortices, but equilibrium vortex configurations composed of singly quantized vortices only. 